\title{
A Quantum Statistical Calculation of Nuclear Quadrupole Moment in Metallic Zinc
}

\author{
B C Rai \\ Associate Professor, PG Centre, Department of Physics, College of Commerce, Patna, India \\ (Magadh University, Bodhgaya)
}

\begin{abstract}
Nuclear Quadrupole Moment (NQM) of zinc nucleus in pure metallic environment is calculated using quantum statistically obtained electric field gradient (EFG) tensor at nuclear site and available nuclear quadrupole coupling coefficients in non-interference of atomic shell approximations. Result does not compare well with previous data, and indicates inclusion of additional shell effect in model based NQM calculations.
\end{abstract}

Keywords: Nuclear Quadrupole moment, Zinc metal, Electric field gradient, quantum statistics, p-waves

\section{Introduction}

Zinc is an integral constituent of a large number of enzymes, inorganic and biological molecules. To better understand its catalytic and structural role in such systems, it is suggestive to study both ${ }_{30}^{67} \mathrm{Zn}$ NMR- tensors, chemical shift and EFG tensors. The Zn (II) center in such systems is surrounded in definite way by ligands, as in complexes, and ionic network, as in oxides and aluminates. Periodicity of structure plays vital role in these structures. Similar is the case of metallic zinc, in which ions form hexagonal close packed lattice, and conduction electrons are surrounding them.

Limitations arise in quantum mechanical calculations of EFG, and hence NQM, due to lack of exact wave functions. Software like Gaussian 98 suite of programs use limited basis sets of electrons, calculations performed at restricted Hartree Fock level and density functional level. Accurate calculation of EFG requires large basis set to encompass core and valence polarization, and often face slow convergence with standard basis sets. On the other hand, qualitatively good basis set for heavy elements are rare. Several approximate approaches are found in literature. We here use a quantum statistical approach essentially of Thomas Fermi model of atoms [1-3] type, and used by Bodenstedt et al[4], but using a different way of lattice summation.

The NQM of zinc in metallic environment is calculated using the EFG and available NQCC data.

\section{Computational Details}

The conduction electrons in zinc fill up the space between spherically symmetric metal ions according to quantum statistics as assumed for free atoms by Thomas and Fermi in statistical atomic model [1]. The assumption does not exhibit the electronic shell effects of interference pattern but is a good approximation for electrostatic potential of atoms used till date. We assume the shell effects in our three dimensional Thomas-Fermi lattice calculations as insignificant as a first approximation and see its effect on calculated values. Here we find a charge distribution exclusively determined by the available volume in phase space of zinc metal. Once the charge distribution is at hand we can calculate EFG, and hence NQM.

There is one to one correspondence between electrons occupying a volume $d V$ in space between ions, and available phase cells in phase space. As conduction electrons can have momentum from zero to Fermi momentum, $p_{f}$, they lie within a sphere of this radius in momentum space. Heisenberg's uncertainty principle and Sackur-Tetrode results show that their charge density may be written, using quantum statistics, as

$$
\rho_{e l}(r) d V=(-e)\left[\frac{4 \pi p_{f}^{3}}{3 h^{3}}\right] \times 2 d V
$$

The factor 2 cares for two spin states of electrons, and a phase cell is of volume $h^{3}$.

The Fermi momentum is derived from total energy of electron in conduction band that is very close to the Fermi energy. Using the potential $\varphi(\boldsymbol{r})$ at position $\boldsymbol{r}$, and the kinetic energy of electron corresponds to Fermi momentum, we get

$$
E_{F}=(-e) \varphi(r)+\frac{p_{f}^{2}}{2 m}
$$

The conduction electronic charge distribution may now be written, using above two equations, as

$$
\rho_{e l}(r)=-\frac{8 \pi e}{3 h^{3}}(2 m)^{\frac{3}{2}}\left[E_{F}+e \varphi(r)\right]^{\frac{3}{2}}
$$

As the unit cell is neutral, the volume integral of the conduction electron charge density over the unit cell must be opposite to the total ionic charge. Let $\mathrm{z}$ e be the ionic charge and there are $\mathrm{k}$ such ions per unit cell. Then

$$
\iiint \rho_{e l}(r) d V=-k z e
$$

The Fermi energy is fixed by this secondary condition.

The total charge density $\rho(\boldsymbol{r})$ and potential $\varphi\left(r^{\prime}\right)$ are related in the Poisson equation formed as 


\section{International Journal of Science and Research (IJSR)}

ISSN (Online): 2319-7064

Index Copernicus Value (2013): 6.14 | Impact Factor (2014): 5.611

$$
\varphi\left(r^{\prime}\right)=\iiint \frac{\rho(r) d V}{4 \pi \epsilon_{0} r}
$$

Here the integration runs over entire volume. Equations (1) and (3) show that to know the charge density, we need potentials, and to know potentials we need the charge density! In cases of such transcendental equations, numerical or graphical methods are used for solutions.

Instead of dividing the crystal volume into infinitesimal volumes $d V$, we divide the unit cell into $N$ small WignerSeitz unit cells, filling up the Bravais unit cell, with their centres arranged to satisfy the symmetry of the Bravais lattice around an ion. Charges in such sites, each of volume $\omega$, are related to their potentials as

$$
q_{i}=-\omega \frac{8 \pi e}{3 h^{3}}(2 m)^{\frac{3}{2}}\left[E_{F}+e \varphi_{i}\right]^{\frac{3}{2}}
$$

Let there be $M_{i}$ sites per unit cell, for i-th non-equivalent type and $\aleph$ such types. Then the cell neutrality condition is expressed as

$$
\sum_{i}^{\kappa-1} M_{i}\left[E_{F}+e \varphi_{i}\right]^{\frac{3}{2}}=-k z e \ldots
$$

The Fermi energy $E_{F}$ is calculated from eq. (5). Iterations give us self-consistent charges as discussed ahead.

The parameters along a- and b- axes of zinc unit cell are divided into 4 equal parts each and c-axis into 8 equal parts, i.e., $\mathrm{N}=128$. In these, a volume of 128 th part of unit cell volume belongs to each site: $\omega=a^{2} c / 128$. Equivalent sphere replaces the sites for computational ease, whose radii are quickly obtained.

In the process we obtain three non-equivalent planes in which lie 14 non-equivalent sites occupying 128 positions in unit cell. If we calculate ionic charge using wave functions of zinc up to radius decided by the procedure, we get unrealistic charge on an ion. To get a realistic value of charge, ionic volume is taken as that containing first nearest electronic sites. These are 12 . The ionic volume is, now, 13 times the old value. For two ions per unit cell, the total number of sites reduces to 104 and non-equivalent sites to 12 .

The ionic charge is obtained by integration of RoothaanHartree- Fock wave function of zinc[5] over an effective ionic volume determined by the sub-lattice construction. We get

\section{$\mathrm{Z}=2.348$}

This charge is used to get conduction electron charge distribution by iterations. We need potentials in the process. These are written as fast converging series in direct and reciprocal lattice spaces [6].

Let the field point in the $\lambda=0$-unit cell be $\boldsymbol{\tau}_{i_{\mu}}$, and be vacant. Then potential is given by

$$
\begin{aligned}
\varnothing\left(\boldsymbol{\tau}_{i_{\mu}}\right)=\left[\sum q_{v} D_{\mu \nu}+\left(-\frac{2 q_{\mu}}{a_{0}}\right)\right. \\
\left.+\sum q_{\nu} R_{\mu \nu}\right] \frac{1}{4 \pi \epsilon_{0}} \ldots
\end{aligned}
$$

Here summation runs over all types of charges. The matrices are obtained by the lattice sums.

$$
D_{\mu \nu}=\sum_{j=1}^{M_{v}} \sum_{\lambda}^{\prime} \frac{\Gamma\left(\frac{1}{2}, \frac{\pi\left|\boldsymbol{T}_{\lambda}+\boldsymbol{\tau}_{j v}-\boldsymbol{\tau}_{i_{\mu}}\right|^{2}}{a_{0}{ }^{2}}\right)}{\Gamma\left(\frac{1}{2}, 0\right)\left|\boldsymbol{T}_{\lambda}+\boldsymbol{\tau}_{j v}-\boldsymbol{\tau}_{i_{\mu}}\right|}
$$

The gamma functions are given by

$$
\Gamma(\eta, \xi)=\int_{\xi}^{\infty} \exp (-\xi) \xi^{\eta-1} d \xi
$$

This is also known as incomplete gamma function.

The reciprocal lattice sum is carried over real part of the sum

$R_{\mu \nu}=\frac{1}{\pi \Omega_{0}} \sum_{j_{v}=1}^{M_{v}} \sum_{\lambda}^{\prime} \frac{\exp \left(-2 \pi i K_{\lambda} \cdot\left(\boldsymbol{\tau}_{j v}-\boldsymbol{\tau}_{i \mu}\right)-\pi a_{0}^{2} K_{\lambda}^{2}\right)}{K_{\lambda}^{2}}$

The prime on summation indicates that for $\lambda=0$, one should not have $\boldsymbol{\tau}_{j v}=\boldsymbol{\tau}_{i_{\mu}}$.

We have calculated these matrices for $\mathrm{Zn}$ using FORTRAN.

Using the tabulations and iterations of Newton Raphson, we obtained the charges at 11 electronic non-equivalent sites [Table 1].

The electric field gradient at origin due to point charge $q$ at position $\boldsymbol{r}$ relative to a vacant ionic site taken as origin of co-ordinates in the crystal produces electric field intensity at the origin whose space rate of variation along $c$-axis taken as Z-axis is given by

$V_{z z}=\frac{q}{4 \pi \epsilon_{0}} \frac{3 z^{2}-r^{2}}{r^{5}}$

Let us reformulate it for zinc crystal. Let $N_{i}=n_{i}+t_{i j_{k}}$ denotethei-th component of position vector of nonequivalent charge located at $k$-th position of $j$-th nonequivalent type site in the $n$-th unit cell. The multiplicity per unit cell for a $j$-th non-equivalent site is denoted by $M_{j}$. In this notation system, the position vectors may be written as

$\boldsymbol{r}=\sum N_{i} \boldsymbol{u}_{i}(i=1$ for $\mathrm{x}$-component, 2 for $\mathrm{y}$-component and 3 for z-component).

The vectors $\boldsymbol{u}_{\boldsymbol{i}}$ are the unit vector $\boldsymbol{i}, \boldsymbol{j}$ and $\boldsymbol{k}$. The position vector of a site in zinc crystal is, thus, given by

$$
\boldsymbol{r}=\left(\mathrm{n}_{1}+t_{1 j_{k}}\right) \mathbf{i}+\left(\mathrm{n}_{2}+t_{2 j_{k}}\right) \mathbf{j}+\left(\frac{c}{a}\right)\left(\mathrm{n}_{3}+t_{3 j_{k}}\right) \mathbf{k} .
$$




\section{International Journal of Science and Research (IJSR) \\ ISSN (Online): 2319-7064}

Index Copernicus Value (2013): 6.14 | Impact Factor (2014): 5.611

With these notations, and Z-axis as c-axis of crystal, the EFG may be written as

$$
V_{z z}=(1-R) \sum_{j=1}^{11} q_{j} B_{j}+\left(1-\gamma_{\infty}\right) q_{12} B_{12} \ldots
$$

Using lattice summations, we obtain the values of quantizes in this equation; these are shown in table 1. The value of $\mathrm{R}$ is -9.00 [7], and the value of the Sternheimer anti-shielding factor is -12.31 for ionic zinc [8]. With these values the electronic EFG (11 non-equivalent sites) turns out to be positive in sign and equals $4.349 a u$, and ionic EFG (12th non-equivalent sites) bears negative sign and equals $-4.465 \mathrm{au}$. The total $\mathrm{EFG}$ is, thus, equal to $0.116 a u$.

The measured quantities are nuclear quadrupole coupling constant (NQCC) and asymmetry parameter in EFG, that appear in the expression

$$
v_{Q}=e Q \frac{V_{z z}}{h} \ldots
$$

with $V_{z z}$ as principal component of EFG tensor along spin quantization axis, the Z-axis, and asymmetry parameter $\eta=\left(V_{x x}-V_{y y}\right) / V_{z z}$. The recent NQCC value for zinc is 12.25 MHz [9], which we use in equation (9) to get a quadrupole moment of zinc in metallic environment as

$$
\begin{gathered}
Q(b)=\frac{v_{Q}}{234.9647 V_{z z}}=\frac{12.35 \mathrm{MHz}}{234.9647 \times(-0.116 a u)} \\
=-0.453 \mathrm{~b}
\end{gathered}
$$

Table 1: Charges and $B_{i}$ in zinc

\begin{tabular}{|c|c|c|}
\hline $\begin{array}{c}\text { Non-equivalent } \\
\text { Sites }\end{array}$ & $\begin{array}{c}\text { Charge in units } \\
\text { of e }\end{array}$ & $\begin{array}{c}B_{j} \text { in units of } 1.44 \times 10^{21} \\
\mathrm{~V}^{2} \mathrm{~m}^{2}\end{array}$ \\
\hline 1 & -0.07077082 & 24.4810400 \\
\hline 2 & -0.05191937 & -32.4921300 \\
\hline 3 & -0.03366279 & -27.0163700 \\
\hline 4 & -0.03007849 & -9.4726350 \\
\hline 5 & -0.04888698 & -45.3905700 \\
\hline 6 & -0.05059905 & -47.5129100 \\
\hline 7 & -0.05508910 & -50.134880 \\
\hline 8 & -0.03683521 & 37.1529200 \\
\hline 9 & -0.04173968 & 92.2081600 \\
\hline 10 & -0.03213683 & 36.6918800 \\
\hline 11 & -0.02071887 & 1.8778090 \\
\hline 12 (ion) & 2.348 & -0.9642023 \\
\hline
\end{tabular}

\section{Discussion}

The result based on quantum statistical approach taken here is matching in sign of EFG and quadrupole moment of nucleus and thus correctly gives the nuclear shape, but the numerical value of quadrupole moment is thrice the known value of $0.151 \mathrm{~b}$ for zinc $[9,10]$. This suggests that quantum statistics of Thomas Fermi model is not able to give correct $\mathrm{EFG}$ of $\mathrm{Zn}$. The results of electronic and ionic EFG are in clear contrast with values on page 17 of ref[2]. They correspond to Raghavan's universal correlation of about 1 , and not about 3 [11]. The result may be improved by further work such as additional consideration of $\mathrm{p}$-wave electronic contributions of electrons surrounding zinc nucleus and showing local effect on EFG, and hence NQM of zinc. The shell effects are not featured in ThomasFermi type of approach.

It may be mentioned here that the computations for tetragonal close packed structures like Indium has compared well using essentially the same procedure [12$14]$.

\section{Conclusion}

The quantum statistical way of charge distribution, exclusively determined by the available volume in phase space of hep lattice of zinc metal, which facilitated computation of EFG, and hence NQM, gives correct signs of electronic and ionic EFGs but magnitudes are a bit smaller. This results in enhanced value of calculated nuclear quadrupole moment. The initial approximation of assuming shell effects insignificant is not correct at least in zinc, though such an approach was a success in tcp metal Indium. Additional contribution of $\mathrm{p}$-wave of $\mathrm{Zn}$ to $\mathrm{EFG}$ need be considered before actual computation of quadrupole moment of zinc nucleus in hexagonal close packed metallic environment.

\section{References}

[1] Thomas, L. H.: Proc. Camb. Philos. Soc. 23, 542(1927)

[2] Fermi, E.: Z. Phys. 48, 73(1928); ibid 49, 550(1928)

[3] Dirac, P. A. M.: Proc. Camb. Philos. Soc. 26, 276(1930)

[4] Bodenstedt, E., Perscheid, B., Nagel, S.: Phy BCondensed Matter 63, 9-24(1986)

[5] Clementi, E., Roetti, C.: At. Data Nucl. Data Tables 14, 234(1974)

[6] Ewald, P. P.: Ann. Phys. (Lipzig) 64,253(1921)

[7] Pattnaik, P. C.,Thompson, M. P., Das, T. P.: Phys. Rev. B 16,5390(1977)

[8] Feioc, F. D., Johnson, W R. : Phys. Rev. 187, 39(1969)

[9] Hass, H., Correia, J. G.: Hypfine Interact, 198, 133137(2010)

[10]P.Pyykkö, Mol. Phys. 99, 1617(2001).

[11] Raghavan, R. S., Kaufmann, E.N., Raghvan, P.: Phys.Rev.Lett.34, 1280(1975).

[12] Rai,B.C., Kumar A,International Journal of Pure and Applied Physics. Volume 9, Number 1, pp. 5156(2013)

[13] Rai,B.C.,International Journal of Physics and Applications, Volume 6, Number 1, pp. 81-86(2014)

[14]Rai, B C ,International Journal of Applied Physics, Volume 4, Number 1, pp. 21-28(2014) 